\title{
Including a General practice endorsement letter with the testing kit in the Bowel Cancer Screening Programme: results of a cluster randomised trial
}

\author{
Amanda J. Cross ${ }^{1}$, Jonathan Myles ${ }^{2}$, Paul Greliak ${ }^{1}$, Allan Hackshaw ${ }^{3}$, Stephen Halloran ${ }^{4,10}$, Sally C \\ Benton $^{4,11}$, Caroline Addison ${ }^{5}$, Caroline Chapman $^{6}$, Natasha Djedovic ${ }^{7}$, Stephen Smith ${ }^{8}$, Christian von \\ Wagner $^{9}$, Stephen W. Duffy*2, Rosalind Raine*9 \\ ${ }^{1}$ Department of Surgery and Cancer, Imperial College London, London, W2 1PG \\ 2 Wolfson Institute for Preventive Medicine, Barts and the London School of Medicine and Dentistry, Queen Mary University \\ of London, London, EC1M 6BQ \\ 6BT \\ ${ }^{3}$ Cancer Research UK and UCL Cancer Trials Centre, Cancer Institute, University College London, London W1T 4TJ \\ ${ }^{4}$ Bowel Cancer Screening Southern Programme Hub, Surrey Research Park, Priestly Road, Guildford, GU2 7YS \\ ${ }^{5}$ Bowel Cancer Screening North East Programme Hub, Queen Elizabeth Hospital, Gateshead, NE9 6SX \\ ${ }^{6}$ Bowel Cancer Screening Eastern Programme Hub, University of Nottingham, Nottingham, NG7 2UH \\ ${ }^{7}$ Bowel Cancer Screening London Programme Hub, Northwick Park \& St Mark's Hospitals, Harrow, HA1 3UJ \\ ${ }^{8}$ Bowel Cancer Screening Midlands \& North West Programme Hub, Hospital of St Cross, Rugby, CV22 5PX \\ ${ }^{9}$ Department of Applied Health Research, University College London, London WC1E \\ ${ }^{10}$ Department of Biomedical Sciences, University of Surrey, Guildford, GU2 7WG \\ ${ }^{11}$ Berkshire and Surrey Pathology Services, Royal Surrey County Hospital, Guildford, UK
}

*Contributed equally to this work 


\section{Abstract}

Objectives: To evaluate the effect of general practice endorsement accompanying the screening kit rather than with the invitation letter on participation in the NHS Bowel Cancer Screening Programme and on the socioeconomic gradient in participation in the Programme.

Methods: The NHS Bowel Cancer Screening Programme in England is delivered via five regional hubs. In early 2016, we carried out a cluster-randomised trial, with hub-day of invitation as the randomisation unit. We randomised 150 hub-days of invitation to the intervention group, GP endorsement on the letter accompanying the guaiac faecal occult blood testing kit (75 hub-days, 197,366 individuals) or control, usual letter ( 75 hub-days, 197,476 individuals). The endpoint was participation, defined as return of a valid kit within 18 weeks of initial invitation. Because of the cluster randomisation, data were analysed by a hierarchical logistic regression, allowing a random effect for date of invitation. Socioeconomic status was represented by the index of multiple deprivation (IMD).

Results: Participation was $59.4 \%$ in the intervention group and $58.7 \%$ in the control group, a significant difference $(p=0.04)$. There was no heterogeneity of the effect of intervention by IMD. We found that there was some confounding between date and screening episode order (first or subsequent screen). This in turn may have induced confounding with age and slightly diluted the result.

Conclusions: General Practice Endorsement induces a modest increase in participation in bowel cancer screening, but does not affect the socioeconomic gradient. When considering cluster randomisation as a research method, careful scrutiny of potential confounding is indicated in advance if possible and in analysis otherwise. 


\section{Introduction}

In England, the NHS Bowel Cancer Screening Programme (BCSP) has been offering faecal occult blood testing (FOBt) since 2006 [1]. The programme has the lowest participation of the three cancer screening programmes, at around $60 \%$, compared to participation rates of around $70 \%$ in screening programmes for breast cancer and $80 \%$ for those for cervical cancer. [2,3]. Participation is especially low in deprived populations, decreasing to $35 \%$ in the most deprived quintile of the population [2]. Several interventions have been observed to improve screening participation, including general practitioner (GP) endorsement, varying reminder strategies and advance notification, although there is little published work showing a successful reduction in socioeconomic gradients in screening participation [4]. In a series of randomised trials, the ASCEND programme investigated four interventions aimed at reducing this socioeconomic gradient in bowel cancer screening uptake (randomisations occurred between 2012 and 2013):

- a supplementary 'Gist' leaflet very briefly summarising the key information about FOBt screening;

- a supplementary narrative leaflet incorporating individual patients' stories;

- an enhanced reminder letter for those not returning a completed FOBt kit within 4 weeks;

- GP endorsement on the initial invitation letter (which does not include the FOBt kit).

The investigators found that an enhanced reminder to those who had not yet returned a kit after 4 weeks increased overall participation by 0.7 percentage points (from $25.1 \%$ using the standard letter to $25.8 \%$ ) and reduced the socioeconomic gradient, and that GP endorsement increased overall participation by $0.7 \%$ percentage points (from 57.5 to $58.2 \%$ ), without significantly altering the socioeconomic gradient [5].

In view of the results of ASCEND, an additional trial was carried out - ASCEND2, to evaluate the effect of GP endorsement on the letter which accompanies the FOBt kit, this letter and kit being subsequent to the original invitation. At the time of the trial the English BCSP used a guaiac faecal occult blood test which was replaced with an immunochemical faecal occult blood test in 2019.

The aim was to increase screening uptake and reduce differences in uptake by socioeconomic status. This paper reports on the results of ASCEND2. We also flag up a warning on the design of large-scale cluster randomised trials.

\section{Methods}

The NHS BCSP in England is run by five regional hubs. Each hub is responsible for invitation, testing and recall for further investigation following a positive test. The basic timeline for a screening episode is as follows:

1. Week 0: first, an invitation letter is sent making the offer of screening, giving notification that an FOBt kit will arrive in around a week's time, and enclosing information on the pros and cons of FOBt screening.

2. Week 1: the FOBt kit is sent, with instructions for its use.

3. Week 5: if the kit has not been returned within four weeks of its issue, a reminder letter is sent.

4. Receipt of completed kit or week 17: screening episode closed. 
As part of the original ASCEND trials [5], a Primary Care Advisory Group developed text that could be easily incorporated into the standard BCSP invitation as there is space on the letters to mention the support of the individual's GP practice. The group created a GP endorsement that was programmed to appear as a banner across the initial invitation letter linking each individual's letter to their registered GP practice.

The study design of ASCEND2 was identical to that of the previous ASCEND GP endorsement trial except that the intervention banner of the GP practice was now included on the letter with the screening kit rather than as part of the initial invitation letter, and ASCEND2 contained a larger number of subjects. An example letter is included in the supplementary appendix. The cost of including the letter with the kit was the same as including it with the invitation letter, so no additional costs were incurred relative to that of the GP endorsement arm of the ASCEND trial.

We randomised by clusters by day within hubs (hub-days) rather than individuals, as the hubs anticipated administrative difficulties in individual randomisation. Although blinding of hubs was not possible, there was no direct contact with participants.

The primary aim of the study was to test the hypothesis that participation, in terms of percentage of invitees adequately screened was the same in GP Endorsement arm was the same as in the standard invitation arm, against the alternative that participation increases in the GP endorsement arm.

\section{Study population}

Consent had been sought from all general practices in England $(N=8,142)$ in collaboration with a Primary Care Advisory Group and the Health \& Social Care Information Centre (HSClC, now NHS Digital), by sending invitations to be part of the ASCEND trials, followed by reminders at 4 and 8 weeks. Permission to link the GP practice address to the invitation was granted by $80 \%$ of practices $(N=6,480)$. Eighty-one practices from three clinical commissioning groups in the Leeds area that had previously participated in ASCEND were excluded from the ASCEND2 trial to avoid conflicting with another bowel screening uptake trial.

Men and women aged 60-74 years from the GP practices included who are routinely invited for FOBt screening in the BCSP were randomised in equal proportions (clustered by date of invitation, stratified by BCSP hub) to receive a GP practice endorsement letter with their FOBt kit on randomly selected days within a pre-specified time-period, or the control group who received the kit with the standard letter without the GP endorsement banner. Randomisation was carried out via the Bowel Cancer Screening System (BCSS) on which the necessary modifications had to be programmed to enable the system to generate the intervention letters for patients at participating practices. Within each hub, we randomised the working days between $1^{\text {st }}$ February 2016 and $11^{\text {th }}$ March 2016.

Consent forms were not applicable in this study as the intervention took place as part of the subject's usual communication from the BCSP. As there was no direct contact, individuals would have been unaware of participation unless they had been invited on a previous occasion and recalled the content of the invitation or if a household member had received an invitation during the study period that contained different information. The activities of the BCSP are covered under an existing Confidentiality Advisory Group (CAG) Section 251 approval with regard to the handling of patient-identifiable data (Ref: PIAG 1-08(a)/2003). Ethical approval for ASCEND2 was obtained from 
the East Midlands - Leicester Central Research Ethics Committee (15/EM/0561). The ASCEND2 trial is registered with the ISRCTN (http://www.isrctn.com/ISRCTN11660314).

\section{Outcomes}

We used the English Index of Multiple Deprivation (IMD) score to measure socioeconomic status, because of its established representation of deprivation by income, education, housing, health, environment, employment and crime [6] and its demonstrated ability to explain area-level variation in bowel cancer screening uptake [2]. IMD is freely available and widely accepted and used, enabling direct comparison of our results with other studies. The postcode variable was substituted with an IMD score by HSCIC. IMD was applied using the geographic unit of Lower Super Output Area (LSOA) level. There are 32,844 LSOAs in England, each covering about 1,500 individuals. Each individual's postcode was linked to the relevant LSOA with the corresponding IMD scores grouped into quintiles of their national distributions.

The primary outcome was the proportion of people in each IMD quintile returning an adequate FOBt within 18 weeks of being sent their initial invitation letter; this time period coincides with when the BCSS closes an episode due to non-response. An adequate FOBt in this study was defined as reaching a definitive FOBt outcome of either a 'Normal' (no further clinical investigation required) or 'Abnormal' (referral for colonoscopy).

A pseudonymised dataset from the BCSS was provided by HSCIC. Age, sex and the type of screening episode (whether individuals were being invited for the first time, being sent a biennial invitation having previously not responded, or being sent a biennial invitation having been screened before) were obtained from the BCSS database.

\section{Sample size}

To calculate the required study size, we needed to take into account: (1) the size of the effect/interaction we wish to detect; (2) the anticipated distribution of socioeconomic status and the expected participation by socioeconomic status in the control arm and by screening round; (3) the proportion of GP practices agreeing to be included in the trial; (4) a variance inflation factor to account for the cluster (hub-day) randomisation; and (5) the size of the effect/interaction by screening round.

We assumed an interaction such that in the least deprived quintile, there was a $1 \%$ increase in participation with the intervention, in the second least deprived a $1.5 \%$ increase, and so on, with a $3 \%$ increase in the most deprived quintile. This would give an average $2 \%$ increase with the intervention, lower than the $3 \%$ observed in ASCEND [5]. The distribution of IMD from the GP endorsement on the initial invitation letter in ASCEND [5] by completion rates in the control group are shown in Table 1. We anticipated these rates in the control group of ASCEND2 and the intervention participation rates in the final column of Table 1.

We calculated the required sample size using the method of Brentnall et al [7]. To obtain 90\% power to detect the interaction as significant at $5 \%$ level with two-sided testing, in an individually randomised study, based on the control group IMD distribution and participation rates in Table 1, we would require 11,714 individuals in each arm. In the original ASCEND trial, $80 \%$ of the GPs approached agreed to participate in the GP endorsement trial. We therefore inflated the required size for the current trial to $14,643(=11,714 / 0.8)$ subjects per trial arm. We also estimated a variance inflation factor for cluster randomisation of 1.7 , but results post hoc suggest that a larger inflation would be more appropriate. We therefore used an inflation factor of 2.5 for the trial reported on here, giving a total study size of 36,608 per arm, a total number of 73,216 individuals randomised over all five hubs. Assuming approximately 3,000 kits were sent per hub-day, this indicates that we 
should randomise 25 hub-days in total. To cope with any plausible failures of our assumptions, and to allow for subgroup analyses, we randomised 30 days in each hub, completing trial accrual from the five hubs in six working weeks with a target population of 450,000 subjects if our assumption about numbers invited per hub-day were correct.

\section{Statistical analysis}

Data were first analysed by a fixed effects logistic regression and a simple chi-squared test to compare participation rates between the two trial arms. Thereafter a formal analysis, taking account of the cluster randomisation, was carried out using a hierarchical logistic regression model, with a random effect for date, and fixed effects for hub, trial arm IMD quintile, gender and screening round, with estimation by Markov Chain Monte Carlo [8]. The endpoint was participation, defined as returning a valid completed kit by week 17 , that is, 18 weeks after the initiation invitation letter, as noted above. We used the same analysis to examine the secondary endpoints time taken to return the kit, rate of spoilt kits, and rate of kit not delivered.

\section{Results}

Figure 1 shows the flow of participants through the trial. After exclusion of subjects for which GP consent to endorsement was not in place by the appropriate date, there were 395,014 subjects randomised, and after exclusion of the $0.04 \%$ of subjects erroneously included from nonparticipating practices, there were 394,842 subjects, 197,366 randomised to the intervention and 197,476 to the control arm. These represented 150 randomisation units (hub-days), 75 intervention and 75 control. Table 2 shows the basic characteristics of the study population in the intervention and control arms; these were evenly balanced between the groups except for a slightly lower percentage of first-time invitees in the intervention arm (14.5\%, versus $15.7 \%$ in the control arm).

The effect of intervention on being adequately screened within 18 weeks of initial invitation is shown in Table 3. Participation was slightly higher in the intervention arm at $59.4 \%$, compared to $58.7 \%$ in the control arm ( 0.7 percentage point increase). With a conventional analysis at individual level, this would be highly significant $(\mathrm{OR}=1.03,95 \% \mathrm{Cl} 1.01-1.05, \mathrm{p}<0.0001)$. However, when we took account of the cluster randomisation in a hierarchical logistic regression model, the statistical significance was considerably attenuated, at $p=0.04$, and with an odds ratio of $1.02(95 \% \mathrm{Cl} 1.00$ 1.04).

Table 4 shows the results by IMD quintile of invitee, and by hub. There was no significant relationship with the size of the effect of intervention by IMD quintile, nor did the effect vary significantly by age or sex (data not shown). However, there was a noticeable difference between hubs with respect to effect size. In the Midlands and North Western Hub, there was an effect in the opposite direction to the other hubs, with individuals in the control group more likely to participate than those in the intervention group. In the hierarchical model, there was a significant heterogeneity of the effect of the intervention by hub $(p<0.001)$, which lost its significance when adjusted for screening round $(p=0.2)$

There was a systematic difference between dates with respect to screen type (first/subsequent) and therefore also with respect to age. Table 5 shows screen type by date. It can be seen that generally less than $20 \%$ of episodes were first time invitations. However, for $25^{\text {th }}$ February, $47 \%$ were firsttime invitations, for the $26^{\text {th }}$ February $80 \%$ were first time, for $29^{\text {th }}$ February, $49 \%$, and for $1^{\text {st }}$ March, $67 \%$. The same pattern was seen for all five hubs, and this had implications for the age of the invitees: first time invitations were predominantly aged 59-64 years around the end of February and 
the beginning of March. Since first screens are associated with lower participation, the randomisation unit may have had some prior confounding with the outcome.

The pattern of median time in days to return of kit against IMD Quintile was exactly the same in each treatment arm - 14 days in the four lowest quintiles of deprivation and 13 in the highest. The rate of spoilt kits rose from $0.6 \%$ in the least deprived quintile to $1.3 \%$ in the most, and the rate of nondelivered kits rose from $32.2 \%$ to $55.8 \%$. Bayesian multilevel modelling similar to that carried out for participation showed that intervention had no significant effect on either rate, nor did the intervention effect vary significantly by IMD quintile.

\section{Discussion}

In this cluster-randomised trial with almost 400,000 subjects, we found at best a modest effect of GP practice endorsement with the FOBt kit on participation, with $59.4 \%$ participation in the intervention group and $58.7 \%$ in the control group. This was consistent with the result of the first ASCEND trial, of GP endorsement on the initial invitation letter, which showed a participation proportion of $58.2 \%$ compared to $57.5 \%$ in the control group [9]. We had hypothesised that the increased uptake due to the intervention might have a different effect by socioeconomic status. However, there was no significant heterogeneity of the effect by IMD quintile.

We observed a systematic difference between days with respect to the invited population. Around the end of February and the beginning of March 2016, a higher proportion of younger and first time potential screenees were invited. Given the lower participation at first round observed both here and elsewhere [10], this constitutes a confounding risk.

While the cluster-randomisation option is attractive in very large-scale population trials in prevention and screening, the results here give a salutary warning that it is not without hidden costs. The use of a mixed model analysis allows for additional variation to pure sampling error among clusters, but does not adequately adjust for the systematic difference observed here. Before choosing such a design, careful advance checking of assumptions with respect to both random and systematic differences among clusters is essential. Heterogeneities among randomisation units may inform further stratification, exclusion policy or indeed a decision not to use the cluster randomisation design or define randomisation units differently.

We observed a difference between hubs with respect to effect size. In the Midlands and North Western Hub, there was an effect in the opposite direction to overall, with the control group having higher participation than the intervention group. This is likely due to the fact that in the Midlands and North Western Hub, the three days dominated by the first invitation round and the 59-64 year age group were all randomised to the intervention arm, conferring a lower participation in the intervention group. The result from all four other hubs (all of whom happened to have the crucial dates shared between intervention and control) combined gave a $1.6 \%$ increase in participation with intervention (61.4\% vs $59.8 \%$ ). This suggests that the true effect may be slightly larger than the primary result reported here, but even taking into account the potential confounding issue of date of invitation, the effect of the intervention on participation remained modest, and rather smaller than the effect of primary care endorsement seen in other contexts [4,5,11]. As noted above, however, it was consistent with the results of the original ASCEND trial of GP endorsement [9].

Thus, in conclusion, two observations can be made. The first is that the effect of GP practice endorsement on the letter accompanying the bowel screening kit is modest (although from above, it may be slightly greater than observed overall in this study) and shows no sign of variation with socioeconomic status. Neither does placing the GP endorsement in a different letter closer to when people are asked to actually participate make a substantial difference. Second, cluster 
randomisation should only be used after careful checking of assumptions with respect to differences among clusters.

Finally, notwithstanding the small effects observed in GP endosrement trials, the BCSP now routinely places the GP banner on the invitation and test kit letters. This banner now accompanies a more user-friendly test kit which only requires a single sample and is associated with an improvement in uptake [12]. It is possible that small modifications like a GP Endorsement will have a stronger impact after having removed an important practical obstacle (the need to sample take samples from three consecutive bowel motions).

\section{Acknowledgements}

ASCEND2 was originally conceived by our dear colleagues Professor Wendy Atkin, who died in 2018, and Professor Jane Wardle, who died in 2015. The study was led by Professor Atkin until her death. The trial was funded by Cancer Research UK awards (references C8171/A16894; C53889/A25004; and C1418/A14134). Professor Stephen Duffy and Dr Jonathan Myles participated in this study funded by the National Institute for Health Research (NIHR) Policy Research Programme, conducted through the Policy Research Unit in Cancer Awareness, Screening and Early Diagnosis, 106/0001. Professor Rosalind Raine is partly funded by NIHR ARC North Thames. The views expressed are those of the author(s) and not necessarily those of the NIHR or the Department of Health and Social Care.

\section{References}

1. Rajasekhar PT, Clifford GM, Lee TJW, Rutter MD, Waddup G, Ritchie M, Nylander D, Painter J, Singh J, Ward I, Dempsey N, Bowes J, Handley G, Henry J, Rees CJ. Bowel cancer screening is safe, detects earlier stage cancer and adenomas in $50 \%$ of cases: experience of the prevalent round of screening from two first wave centres in the North East of England. Frontline Gastroenterol. 2012 Jan;3(1):10-15

2. von Wagner C, Baio G, Raine R, Snowball J, Morris S, Atkin W, Obichere A, Handley G, Logan RF, Rainbow S, Smith S, Halloran S, Wardle J (2011) Inequalities in participation in an organized national colorectal cancer screening programme: results from the first 2.6 million invitations in England. Int J Epidemiol 40(3): 712-718

3. McCowan C, McSkimming P, Papworth R, Kotzur M, McConnachie A, Macdonald S, Wyke S, Crighton E, Campbell C, Weller D, Steele RJC, Robb KA. Comparing uptake across breast, cervical and bowel screening at an individual level: a retrospective cohort study. $\mathrm{Br} J$ Cancer. 2019 Oct;121(8):710-714

4. Duffy SW, Myles JP, Maroni R, Mohammad A. Rapid review of evaluation of interventions to improve participation in cancer screening services. J Med Screen 2017 Sep;24(3):127-145

5. Wardle J, von Wagner C, Kralj-Hans I, Halloran SP, Smith SG, McGregor LM, Vart G, Howe R, Snowball J, Handley G, Logan RF, Rainbow S, Smith S, Thomas MC, Counsell N, Morris S, Duffy SW, Hackshaw A, Moss S, Atkin W, Raine R. Effects of evidence-based strategies to reduce the socioeconomic gradient of uptake in the English NHS Bowel Cancer Screening Programme (ASCEND): four cluster-randomised controlled trials. Lancet. 2016 Feb 20;387(10020):751-9.

6. https://assets.publishing.service.gov.uk/government/uploads/system/uploads/attachment data/file/464430/English Index of Multiple Deprivation 2015 - Guidance.pdf

7. Brentnall AR, Duffy SW, Baio G, Raine R. Strategy for power calculation for interactions: application to a trial of interventions to improve uptake of bowel cancer screening. Contemp Clin Trials 2012; 33: 213-7

8. Gelman A, Carlin JB, Stern HS, Rubin DB. Bayesian data analysis. Texts in statistical science series. Boca Raton: Chapman \& Hall/CRC; 2004.

9. Raine R, Duffy SW, Wardle J, Solmi F, Morris S, Howe R, Kralj-Hans I, Snowball J, Counsell N, Moss S, Hackshaw A, von Wagner C, Vart G, McGregor LM, Smith SG, Halloran S, Handley G, 
Logan RF, Rainbow S, Smith S, Thomas MC, Atkin W. Impact of general practice endorsement on the social gradient in uptake in bowel cancer screening. Br J Cancer. 2016 ;114:321-6

10. Lo SH, Halloran S, Snowball J, Seaman H, Wardle J, von Wagner C. Colorectal cancer screening uptake over three biennial invitation rounds in the English bowel cancer screening programme. Gut. $2015 \mathrm{Feb} ; 64(2): 282-91$

11. Benton SC, Butler $P$, Allen $K$, et al. GP participation in increasing uptake in a national bowel cancer screening programme: the PEARL project. Br J Cancer 2017; 116: 1551-1557

12. Moss S, Mathews C, Day TJ, et al. Increased uptake and improved outcomes of bowel cancer screening with a faecal immunochemical test: results from a pilot study within the national screening programme in England. Gut. 2017;66(9):1631-1644. doi:10.1136/gutjnl-2015310691 
Table 1. Observed socioeconomic status distribution and control group completion rates from ASCEND GP endorsement trial $(n=133,449)[5]$ and estimated completion rate in the intervention group of ASCEND2. Completion rate is the percentage of individuals who returned a valid FOBt kit by week 17.

\begin{tabular}{lccc}
\hline $\begin{array}{l}\text { Socioeconomic } \\
\text { status (national } \\
\text { IMD quintile) }\end{array}$ & $\begin{array}{l}\text { Control group: } \\
\text { percentage in } \\
\text { each quintile } \\
\text { from ASCEND [5] }\end{array}$ & $\begin{array}{l}\text { Control group } \\
\text { completion } \\
\text { percent from } \\
\text { ASCEND [5] }\end{array}$ & $\begin{array}{l}\text { Anticipated } \\
\text { intervention } \\
\text { group completion } \\
\text { percent in } \\
\text { ASCEND2 }\end{array}$ \\
\hline 1 (least deprived) & 23.4 & 66.0 & 67.0 \\
2 & 23.4 & 62.6 & 64.1 \\
3 & 21.0 & 58.0 & 60.0 \\
4 & 17.2 & 51.5 & 54.0 \\
5 (most deprived) & 14.6 & 42.6 & 45.6 \\
\hline
\end{tabular}


Table 2. Basic description of the study population

\begin{tabular}{|c|c|c|c|c|}
\hline \multirow[t]{2}{*}{ Factor/category } & \multicolumn{2}{|c|}{$\begin{array}{c}\text { Intervention } \\
\mathrm{N}=197,366\end{array}$} & \multicolumn{2}{|c|}{$\begin{array}{c}\text { Control } \\
\mathrm{N}=197,476\end{array}$} \\
\hline & median & (range) & median & (range) \\
\hline Age at invite (in years) & 66 & $(59.0-74.0)$ & 66 & $(59.0-74.0)$ \\
\hline \multirow[t]{2}{*}{ IMD Deprivation score } & 14.8 & $(0.6-87.8)$ & 14.7 & $(0.6-87.8)$ \\
\hline & $\%$ & $\mathrm{~N}$ & $\%$ & $\mathrm{~N}$ \\
\hline \multicolumn{5}{|l|}{ Sex } \\
\hline Female & 50.6 & 99,852 & 50.7 & 100,054 \\
\hline Male & 49.4 & 97,514 & 49.3 & 97,422 \\
\hline \multicolumn{5}{|l|}{ Socioeconomic score quintile } \\
\hline Least deprived (0-8.49) & 23.0 & 45,415 & 22.9 & 45,201 \\
\hline 2nd quintile (8.50-13.79) & 23.2 & 45,884 & 23.3 & 46,018 \\
\hline 3rd quintile (13.80-21.35) & 21.5 & 42,348 & 21.2 & 41,767 \\
\hline 4th quintile (21.36-34.17) & 17.1 & 33,666 & 17.2 & 34,001 \\
\hline Most Deprived (34.18-87.80) & 14.4 & 28,363 & 14.6 & 28,785 \\
\hline Missing & 0.9 & 1,690 & 0.9 & 1,704 \\
\hline \multicolumn{5}{|l|}{ Hub } \\
\hline Midland \& North West & 23.9 & 47,150 & 23.8 & 46,981 \\
\hline Southern & 30 & 59,121 & 30 & 59,260 \\
\hline London & 9.2 & 18,176 & 9.8 & 19,256 \\
\hline North East & 15.7 & 30,888 & 15.6 & 30,899 \\
\hline Eastern & 21.3 & 42,031 & 20.8 & 41,080 \\
\hline \multicolumn{5}{|l|}{ Screening Round } \\
\hline Incident Episodes & 56.9 & 112,358 & 55.9 & 110,326 \\
\hline Prevalent first time invitees & 14.5 & 28,605 & 15.7 & 30,923 \\
\hline Prevalent previous non-responders & 28.6 & 56,403 & 28.5 & 56,227 \\
\hline
\end{tabular}


Table 3. Effect of the intervention on screening participation, as measured by percentage of invitees adequately screened.

\begin{tabular}{lccc}
\hline Trial arm & \multicolumn{2}{c}{ Number (\%) } & Total \\
\hline \multirow{3}{*}{ Intervention } & Adequately screened & Not adequately screened & \\
\cline { 2 - 3 } Control & $117,234(59.4)$ & $80,132(40.6)$ & 197,366 \\
Total & $115,959(58.7)$ & $81,517(41.3)$ & 197,476 \\
\hline
\end{tabular}


Table 4. Effect on participation stratified by IMD and by Hub, as measured by percentage of invitees adequately screened.

\begin{tabular}{|c|c|c|c|c|c|}
\hline \multirow[t]{3}{*}{ Factor/category } & \multicolumn{4}{|c|}{ Participation by trial arm } & \multirow[b]{3}{*}{$\begin{array}{c}\% \\
\text { difference }\end{array}$} \\
\hline & \multicolumn{2}{|c|}{ Intervention } & \multicolumn{2}{|c|}{ Control } & \\
\hline & $\%$ & $\mathrm{~N}$ & $\%$ & $\mathrm{~N}$ & \\
\hline IMD quintile & \multicolumn{2}{|c|}{$N=195,676^{*}$} & \multicolumn{2}{|c|}{$N=195,772 *$} & \\
\hline 1st quintile (least deprived) & 67.9 & 30,849 & 67.1 & 30,314 & 0.8 \\
\hline 2nd quintile & 64.4 & 29,533 & 63.7 & 29,291 & 0.7 \\
\hline 3rd quintile & 60.2 & 25,511 & 60 & 25,075 & 0.2 \\
\hline 4th quintile & 53.2 & 17,906 & 52.5 & 17,835 & 0.7 \\
\hline 5th quintile (most deprived) & 43.8 & 12,425 & 43.2 & 12,432 & 0.6 \\
\hline Hub & \multicolumn{2}{|c|}{$N=197,366$} & \multicolumn{2}{|c|}{$N=197,476$} & \\
\hline Midland and North West & 53.1 & 24,894 & 55.1 & 25,722 & -2.0 \\
\hline Southern & 64.4 & 37,606 & 62.1 & 36,363 & 2.3 \\
\hline London & 48.6 & 8,770 & 47.4 & 9,059 & 1.2 \\
\hline North East & 62.5 & 19,171 & 61.6 & 18,899 & 0.9 \\
\hline Eastern & 61.8 & 25,779 & 61.1 & 24,899 & 0.7 \\
\hline
\end{tabular}

*IMD was missing for $1,690(0.9 \%)$ subjects in the intervention group and $1,704(0.9 \%)$ subjects in the control group. 
Table 5. Episode type by date of kit and letter

\begin{tabular}{|c|c|c|c|}
\hline \multirow[t]{2}{*}{ Date of kit and letter } & \multicolumn{3}{|c|}{ Episode type } \\
\hline & $\begin{array}{c}\text { Incident } \\
\text { or prevalent } \\
\text { previous non- } \\
\text { responder (\%) }\end{array}$ & $\begin{array}{l}\text { Prevalent } 1^{\text {st }} \\
\text { invitation (\%) }\end{array}$ & Total \\
\hline $01 / 02 / 2016$ & $13,832(87)$ & $2,131(13)$ & 15,963 \\
\hline $02 / 02 / 2016$ & $8,970(86)$ & 1,498 (14) & 10,468 \\
\hline 03/02/2016 & $11,284(87)$ & $1,751(13)$ & 13,035 \\
\hline $04 / 02 / 2016$ & $12,177(94)$ & $808(6)$ & 12,985 \\
\hline $05 / 02 / 2016$ & $11,936(92)$ & $1,099(8)$ & 13,035 \\
\hline $08 / 02 / 2016$ & $12,174(93)$ & $926(7)$ & 13,100 \\
\hline 09/02/2016 & $12,382(94)$ & $781(6)$ & 13,163 \\
\hline $10 / 02 / 2016$ & $12,506(95)$ & $696(5)$ & 13,202 \\
\hline $11 / 02 / 2016$ & $12,591(96)$ & $565(4)$ & 13,156 \\
\hline $12 / 02 / 2016$ & $12,572(96)$ & $584(4)$ & 13,156 \\
\hline $15 / 02 / 2016$ & $12,512(95)$ & $591(5)$ & 13,103 \\
\hline $16 / 02 / 2016$ & $12,632(96)$ & $501(4)$ & 13,133 \\
\hline $17 / 02 / 2016$ & $12,710(96)$ & $488(4)$ & 13,198 \\
\hline $18 / 02 / 2016$ & $12,663(97)$ & $428(3)$ & 13,091 \\
\hline $19 / 02 / 2016$ & $12,552(96)$ & $533(4)$ & 13,085 \\
\hline $20 / 02 / 2016$ & $12,766(97)$ & $354(3)$ & 13,120 \\
\hline $23 / 02 / 2016$ & $12,858(97)$ & $388(3)$ & 13,246 \\
\hline $24 / 02 / 2016$ & $12,793(97)$ & $385(3)$ & 13,178 \\
\hline $25 / 02 / 2016$ & $7,010(53)$ & $6,276(47)$ & 13,286 \\
\hline $26 / 02 / 2016$ & $2,626(20)$ & $10,785(80)$ & 13,411 \\
\hline 29/02/2016 & $7,275(51)$ & $7,027(49)$ & 14,302 \\
\hline 01/03/2016 & $8,133(67)$ & $4,004(33)$ & 12,137 \\
\hline $02 / 03 / 2016$ & $10,746(81)$ & $2,445(19)$ & 13,191 \\
\hline 03/03/2016 & $11,285(86)$ & $1,866(14)$ & 13,151 \\
\hline 04/03/2016 & $11,433(87)$ & $1,770(13)$ & 13,203 \\
\hline $07 / 03 / 2016$ & $11,273(86)$ & $1,774(14)$ & 13,047 \\
\hline 08/03/2016 & $10,858(82)$ & $2,313(18)$ & 13,171 \\
\hline 09/03/2016 & $10,500(80)$ & $2,682(20)$ & 13,182 \\
\hline $10 / 03 / 2016$ & $11,442(86)$ & $1,803(14)$ & 13,245 \\
\hline $11 / 03 / 2016$ & $10,823(83)$ & 2,276 (17) & 13,099 \\
\hline
\end{tabular}


Figure 1. - The flow of participants through the trial.

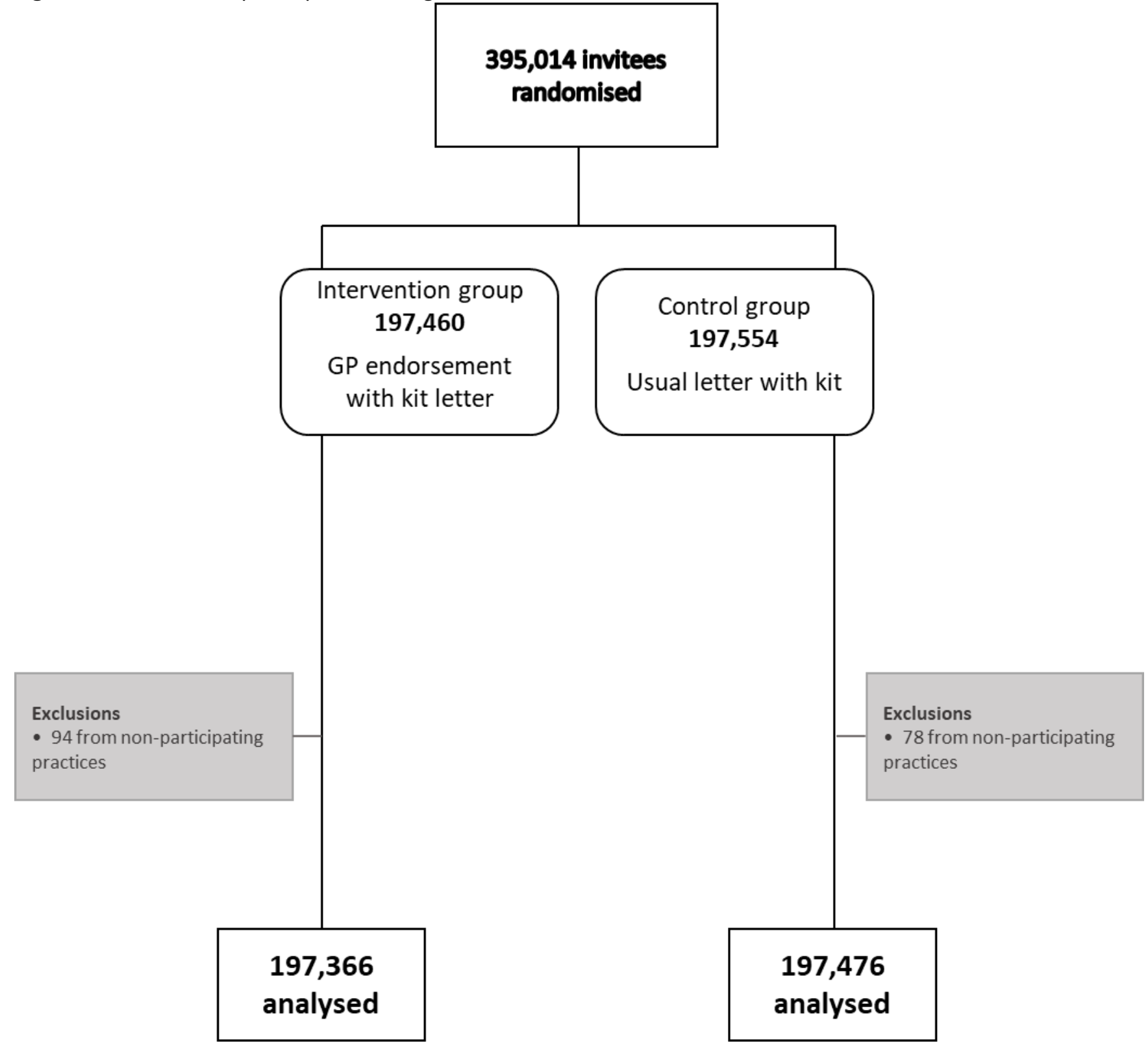

